Supporting Information

for

\title{
Photolytic Living Anionic Ring-Opening Polymerization (ROP) of Silicon-Bridged [1]Ferrocenophanes via an Iron-Cyclopentadienyl Bond Cleavage Mechanism.
}

\author{
Makoto Tanabe and lan Manners* \\ University of Toronto, Department of Chemistry, Davenport Laboratories, \\ 80 St. George Street, Toronto, Ontario M5S 3H6, Canada
}

\section{Experimental Section}

All reactions and manipulations were carried out under an atmosphere of prepurified nitrogen or argon gas (BOC) using common Schlenk techniques or an inert atmosphere glove box (M-Braun). Solvents were dried using a Grubbs-type solvent system $^{1}$ or standard methods followed by sodium/benzophenone distillation. ${ }^{1} \mathrm{H}(300$ or $400 \mathrm{MHz}),{ }^{13} \mathrm{C}\left\{{ }^{1} \mathrm{H}\right\}$ (75.4 or $\left.100.4 \mathrm{MHz}\right)$, and ${ }^{29} \mathrm{Si}\left\{{ }^{1} \mathrm{H}\right\}(79.5 \mathrm{MHz}) \mathrm{NMR}$ spectra were obtained from either Varian Mercury 300 or Varian Unity 400 spectrometers and were referenced either to protic impurities in the solvent $\left({ }^{1} \mathrm{H},{ }^{13} \mathrm{C}\left\{{ }^{1} \mathrm{H}\right\}\right)$ or externally to $\mathrm{SiMe}_{4}\left({ }^{29} \mathrm{Si}\left\{{ }^{1} \mathrm{H}\right\}\right)$ in $\mathrm{C}_{6} \mathrm{D}_{6}$ utilizing a normal pulse sequence. Mass spectra were obtained with the use of a VG 70-250S mass spectrometer operating in Electron Impact (EI) mode. Elemental analyses were performed at the University of Toronto using a Perkin-Elmer 2400 Series CHN Analyzer. Molecular weights were estimated by gel permeation chromatography (GPC) using a Viscotek GPCMax. The GPC instrument contained a column heater, ultrastyragel columns with pore sizes of $10^{3}-10^{5} \AA$, an inline degasser, and a differential refractometer. THF was used as the eluent, with a flow rate of $1.0 \mathrm{~mL} / \mathrm{min}$. Instrument calibration was performed and relative molecular weights were obtained using polystyrene standards purchased from American Polymer Standards. The triple-detector system used (refractive index, light scattering, viscosity) has been shown to provide close to absolute $M_{\mathrm{w}}$ values for PFS homopolymers, and we assume that it provides accurate values of $M_{\mathrm{w}} / M_{\mathrm{n}}{ }^{2}$ Silicon-bridged [1]ferrocenophane 1 of suitably high purity for living anionic polymerization was prepared according to 
the reported procedure. ${ }^{3} \mathrm{Li}\left[\mathrm{C}_{5} \mathrm{H}_{4} \mathrm{Me}\right]$ and $\mathrm{Li}\left[\mathrm{C}_{5} \mathrm{H}_{5}\right]$ were prepared from the reaction of fresh distilled methylcyclopentadiene or cyclopentadiene with a small excess of $n$-BuLi at room temperature in hexane and were purified by washing with hexane. The purity was checked by ${ }^{1} \mathrm{H}$ NMR in $\mathrm{CD}_{3} \mathrm{CN}$. $2.0 \mathrm{M} \mathrm{Na}\left[\mathrm{C}_{5} \mathrm{H}_{5}\right]$ in THF was purchased from Aldrich and was used as received. The photoirradiation experiments used the Pyrex-glass-filtered emission from a $125 \mathrm{~W}$ high pressure $\mathrm{Hg}$ arc lamp (Philips) $(\lambda>$ ca. $300 \mathrm{~nm})$.

Preparation of $\left(\eta^{5}-\mathrm{C}_{5} \mathrm{H}_{4} \mathrm{Me}\right) \mathrm{Fe}\left(\eta^{5}-\mathrm{C}_{5} \mathrm{H}_{4} \mathrm{SiMe}_{2} \mathbf{C}_{5} \mathbf{H}_{5}\right)$ (7) To a solution of sila[1]ferrocenophane $1(1.00 \mathrm{~g}, 4.1 \mathrm{mmol})$ in $30 \mathrm{~mL}$ of THF was added Li[C $\mathrm{C}_{5} \mathrm{H}_{4} \mathrm{Me}$ ] $(1.78 \mathrm{~g}, 20.6 \mathrm{mmol})$ in the absence of light. The solution was irradiated with stirring for $1 \mathrm{~h}$ at room temperature and turned orange in color. $\mathrm{A}{ }^{1} \mathrm{H}$ NMR spectrum of the reaction mixture in acetonitrile- $d_{3}$ showed a mixture of $\left(\eta^{5}-\mathrm{C}_{5} \mathrm{H}_{4} \mathrm{Me}\right) \mathrm{Fe}\left(\eta^{5}-\mathrm{C}_{5} \mathrm{H}_{4} \mathrm{SiMe}_{2} \mathrm{C}_{5} \mathrm{H}_{4} \mathrm{Li}\right)$ and excess of $\mathrm{Li}\left[\mathrm{C}_{5} \mathrm{H}_{4} \mathrm{Me}\right]$. The reaction was terminated with ca. $500 \mu \mathrm{L}$ of degassed water at $0{ }^{\circ} \mathrm{C}$. Any volatile material was completely removed under high vacuum overnight. The product was extracted with hexane and filtered to remove the salt. The hexane solvent was pumped off. The orange oily product was then passed through a $\mathrm{NEt}_{3}$ deactivated alumina column, with cyclohexane as the eluent, to obtain ring-opened product $\left(\eta^{5}-\mathrm{C}_{5} \mathrm{H}_{4} \mathrm{Me}\right) \mathrm{Fe}\left(\eta^{5}-\mathrm{C}_{5} \mathrm{H}_{4} \mathrm{SiMe}_{2} \mathrm{C}_{5} \mathrm{H}_{5}\right)(7)$ in $32 \%$ yield $(427 \mathrm{mg})$. The product contains three regioisomers I, II, and III (vide infra) in 87:11:2 ratios due to hydrogen migration on the $\mathrm{Cp}$ ring. According to a previous report, 5-trimethylsilylcyclopentadiene exhibits fast silyl group and slow hydrogen migration to form three analogous regioisomers in a 90:7:3 ratio. $^{4}$

${ }^{1} \mathrm{H}$ NMR spectrum for crude $\left(\eta^{5}-\mathrm{C}_{5} \mathrm{H}_{4} \mathrm{Me}\right) \mathrm{Fe}\left(\eta^{5}-\mathrm{C}_{5} \mathrm{H}_{4} \mathrm{SiMe}_{2} \mathrm{C}_{5} \mathrm{H}_{4} \mathrm{Li}\right)$ in acetonitrile- $d_{3}$ at $25{ }^{\circ} \mathrm{C}: \delta=5.75\left(\mathrm{~m}, 2 \mathrm{H}, \mathrm{SiC}_{5} H_{4} \mathrm{Li}, J_{\mathrm{HH}}=2.4 \mathrm{~Hz}\right), 5.64(\mathrm{~m}, 2 \mathrm{H}$, $\left.\mathrm{SiC}_{5} H_{4} \mathrm{Li}, J_{\mathrm{HH}}=2.4 \mathrm{~Hz}\right), 5.28\left(\mathrm{~m}, \mathrm{Li}\left[\mathrm{C}_{5} H_{4} \mathrm{Me}\right], J_{\mathrm{HH}}=2.4 \mathrm{~Hz}\right), 5.20\left(\mathrm{~m}, \mathrm{Li}\left[\mathrm{C}_{5} H_{4} \mathrm{Me}\right]\right.$, $\left.J_{\mathrm{HH}}=2.4 \mathrm{~Hz}\right), 4.17\left(\mathrm{t}, 2 \mathrm{H}, \eta^{5}-\mathrm{C}_{5} H_{4} \mathrm{Si}, J_{\mathrm{HH}}=1.8 \mathrm{~Hz}\right), 4.01\left(\mathrm{~m}, 2 \mathrm{H}, \eta^{5}-\mathrm{C}_{5} H_{4} \mathrm{Me}, J_{\mathrm{HH}}=\right.$ $1.7 \mathrm{~Hz}), 4.00\left(\mathrm{~m}, 2 \mathrm{H}, \eta^{5}-\mathrm{C}_{5} H_{4} \mathrm{Me}, J_{\mathrm{HH}}=1.7 \mathrm{~Hz}\right), 3.95\left(\mathrm{t}, 2 \mathrm{H}, \eta^{5}-\mathrm{C}_{5} H_{4} \mathrm{Si}, J_{\mathrm{HH}}=1.8 \mathrm{~Hz}\right)$, $2.00\left(\mathrm{~s}, \mathrm{Li}\left[\mathrm{C}_{5} \mathrm{H}_{4} M e\right]\right), 1.93\left(\mathrm{~s}, \eta^{5}-\mathrm{C}_{5} \mathrm{H}_{4} M e\right), 0.36$ (s, 6H, SiMe $)$.

Data for $\left(\eta^{5}-\mathrm{C}_{5} \mathrm{H}_{4} \mathrm{Me}\right) \mathrm{Fe}\left(\eta^{5}-\mathrm{C}_{5} \mathrm{H}_{4} \mathrm{SiMe}_{2} \mathrm{C}_{5} \mathrm{H}_{5}\right)(7)$ : ${ }^{1} \mathrm{H}$ NMR $\left(\mathrm{C}_{6} \mathrm{D}_{6}, 25{ }^{\circ} \mathrm{C}\right)$ : $6.67\left(\mathrm{br}, \mathrm{SiC}_{5} H_{5}\right), 6.58\left(\mathrm{br}, \mathrm{SiC}_{5} H_{5}\right), 4.82\left(\mathrm{~m}, \eta^{5}-\mathrm{C}_{5} H_{4} \mathrm{Si}\right.$ for III), $4.13\left(\mathrm{t}, 2 \mathrm{H}, \eta^{5}-\mathrm{C}_{5} H_{4} \mathrm{Si}\right.$ 
for I, $\left.J_{\mathrm{HH}}=1.8 \mathrm{~Hz}\right), 4.02\left(\mathrm{~m}, \eta^{5}-\mathrm{C}_{5} H_{4} \mathrm{Si}\right.$ for III), $3.97\left(\mathrm{~m}, \eta^{5}-\mathrm{C}_{5} H_{4} \mathrm{Si}\right.$ for II), $3.92(\mathrm{t}, 2 \mathrm{H}$, $\eta^{5}-\mathrm{C}_{5} H_{4} \mathrm{Si}$ for I, $\left.J_{\mathrm{HH}}=1.8 \mathrm{~Hz}\right), 3.88\left(\mathrm{~m}, 2 \mathrm{H}, \eta^{5}-\mathrm{C}_{5} H_{4} \mathrm{Me}\right.$ for I, $\left.J_{\mathrm{HH}}=1.7 \mathrm{~Hz}\right), 3.87(\mathrm{~m}$, $2 \mathrm{H}, \eta^{5}-\mathrm{C}_{5} H_{4} \mathrm{Me}$ for I, $\left.J_{\mathrm{HH}}=1.7 \mathrm{~Hz}\right), 3.49\left(\mathrm{br}, 1 \mathrm{H}, \mathrm{SiC}_{5} H_{5}, \mathrm{CH}\right.$ signal for I), $2.93(\mathrm{~m}$, $\mathrm{SiC}_{5} H_{5}, \mathrm{CH}_{2}$ signal for II), 2.80 (m, $\mathrm{SiC}_{5} H_{5}, \mathrm{CH}_{2}$ signal for III), 1.83 (s, $\eta^{5}-\mathrm{C}_{5} \mathrm{H}_{4} M e$ for II and III), 1.81 (s, 3H, $\eta^{5}-\mathrm{C}_{5} \mathrm{H}_{4} M e$ for I), 0.47 (s, SiMe 2 for III), 0.43 (s, SiMe for II), 0.13 (s, 6H, SiMe 2 for I). Some signals attributed to minor isomers II and III were not observed or overlapped. ${ }^{29} \mathrm{Si}\left\{{ }^{1} \mathrm{H}\right\}$ NMR $\left(\mathrm{C}_{6} \mathrm{D}_{6}, 25{ }^{\circ} \mathrm{C}\right.$ ): -2.79 (for I), -13.6 (for II), -13.9 (for III). Anal. Calcd. for $\mathrm{C}_{18} \mathrm{H}_{22} \mathrm{FeSi}$ : C, 67.08; H, 6.88. Found: C, 66.69; H, 7.04. MS (70 eV, EI): $m / z(\%): 322(7)\left[M^{+}\right], 257(100)\left[M^{+}-\mathrm{C}_{5} \mathrm{H}_{5}\right]$. High-resolution MS for $\mathrm{C}_{18} \mathrm{H}_{22} \mathrm{FeSi}$ : Calcd. 322.0840, Found 322.0840.
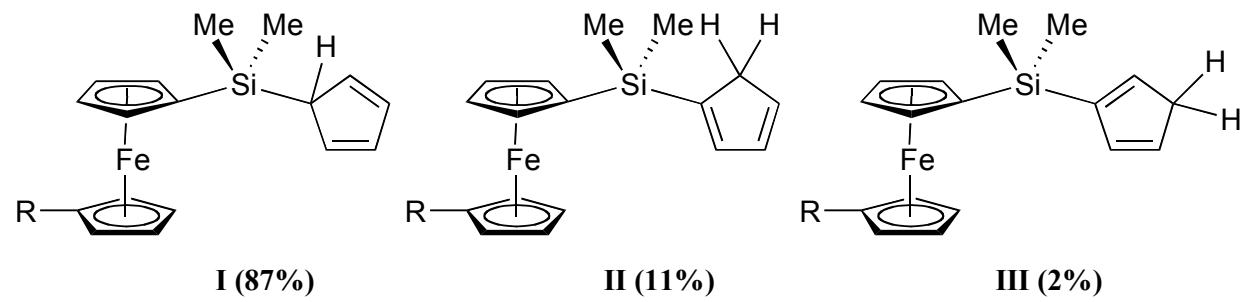

Preparation of low molecular weight polymers 8. To a solution of sila[1]ferrocenophane $1(100 \mathrm{mg}, 0.41 \mathrm{mmol})$ in $1 \mathrm{~mL}$ of THF was added $0.1 \mathrm{M}$ $\mathrm{Li}\left[\mathrm{C}_{5} \mathrm{H}_{4} \mathrm{Me}\right]$ in THF $(0.41 \mathrm{~mL}, 0.041 \mathrm{mmol})$ in the absence of light. The solution was irradiated with stirring for $3 \mathrm{~h}$ at room temperature. The reaction was confirmed to proceed completely by ${ }^{1} \mathrm{H}$ NMR spectrum. The low molecular weight polymer was terminated with a few drops of degassed water, precipitated into methanol, and then dried under high vacuum to obtain a $\mathrm{C}_{5} \mathrm{H}_{4} \mathrm{Me}$ group end-capped oligomer 8a (72 mg, $72 \%$ ). Mass spectrometric analysis of methanol soluble material showed the existence of oligomer $\quad\left[\left(\eta^{5}-\mathrm{C}_{5} \mathrm{H}_{4} \mathrm{Me}\right) \mathrm{Fe}\left(\eta^{5}-\mathrm{C}_{5} \mathrm{H}_{4}\right)\left\{\mathrm{SiMe}_{2} \mathrm{fc}\right\}_{n} \mathrm{SiMe}_{2}\left(\mathrm{C}_{5} \mathrm{H}_{5}\right)\right] \quad(\mathrm{fc} \quad=$ $\left.\left(\eta^{5}-\mathrm{C}_{5} \mathrm{H}_{4}\right) \mathrm{Fe}\left(\eta^{5}-\mathrm{C}_{5} \mathrm{H}_{4}\right)\right)$. MS (EI, $\left.70 \mathrm{eV}\right): m / z=1290(\mathrm{n}=4,<0.01 \%), 1048(\mathrm{n}=3$, $6 \%), 806(\mathrm{n}=2,94 \%), 564(\mathrm{n}=1,8 \%), 322(\mathrm{n}=0,17 \%), 257\left(\mathrm{n}=0,\left[M^{+}-\mathrm{C}_{5} \mathrm{H}_{5}\right]\right.$, 100\%), $243\left(\mathrm{n}=0,\left[M^{+}-\mathrm{C}_{6} \mathrm{H}_{7}\right], 53 \%\right) .{ }^{1} \mathrm{H}$ NMR $\left(\mathrm{C}_{6} \mathrm{D}_{6}, 25{ }^{\circ} \mathrm{C}\right): \delta 6.68\left(\mathrm{SiC}_{5} H_{5}\right), 6.59$ $\left(\mathrm{SiC}_{5} H_{5}\right), 4.27\left(\left(\eta^{5}-\mathrm{C}_{5} H_{4}\right) \mathrm{Si}\left(\eta^{5}-\mathrm{C}_{5} H_{4}\right)\right), 4.21\left(\left(\eta^{5}-\mathrm{C}_{5} H_{4}\right) \mathrm{SiC}_{5} \mathrm{H}_{5}\right), 4.18\left(\eta^{5}-\mathrm{C}_{5} H_{4} \mathrm{Me}\right)$, $4.11\left(\left(\eta^{5}-\mathrm{C}_{5} H_{4}\right) \mathrm{Si}\left(\eta^{5}-\mathrm{C}_{5} H_{4}\right)\right), 4.02\left(\left(\eta^{5}-\mathrm{C}_{5} H_{4}\right) \mathrm{SiC}_{5} \mathrm{H}_{5}\right), 3.96\left(\eta^{5}-\mathrm{C}_{5} H_{4} \mathrm{Me}\right), 3.48\left(\mathrm{SiC}_{5} H_{5}\right.$ for end group structure I), $2.99\left(\mathrm{SiC}_{5} \mathrm{H}_{5}\right.$ for II), $2.82\left(\mathrm{SiC}_{5} \mathrm{H}_{5}\right.$ for III $), 1.85\left(\eta^{5}-\mathrm{C}_{5} \mathrm{H}_{4} M e\right)$, 
$0.55\left(\left(\eta^{5}-\mathrm{C}_{5} \mathrm{H}_{4}\right) \mathrm{Si} \mathrm{Se}_{2}\left(\eta^{5}-\mathrm{C}_{5} \mathrm{H}_{4}\right)\right), 0.30\left(\left(\eta^{5}-\mathrm{C}_{5} \mathrm{H}_{4}\right) \mathrm{Si} M e_{2} \mathrm{C}_{5} \mathrm{H}_{5}\right.$ for II and III $), 0.14$ $\left(\left(\eta^{5}-\mathrm{C}_{5} \mathrm{H}_{4}\right) \mathrm{Si} M e_{2} \mathrm{C}_{5} \mathrm{H}_{5}\right.$ for I). ${ }^{13} \mathrm{C}\left\{{ }^{1} \mathrm{H}\right\}$ NMR $\left(\mathrm{C}_{6} \mathrm{D}_{6}, 25^{\circ} \mathrm{C}\right): \delta 134.0\left(\mathrm{Si}_{5} \mathrm{H}_{5}\right), 131.0$ $\left(\mathrm{Si}_{5} \mathrm{H}_{5}\right), 74.0\left(\eta^{5}-C_{5} \mathrm{H}_{4}\right), 72.1\left(\eta^{5}-C_{5} \mathrm{H}_{4}\right), 70.1\left(\eta^{5}-C_{5} \mathrm{H}_{4} \mathrm{Me}\right), 68.3\left(\eta^{5}-C_{5} \mathrm{H}_{4} \mathrm{Me}\right), 53.6$ $\left(\mathrm{Si}_{5} \mathrm{H}_{5}\right), \quad 15.2 \quad\left(\eta^{5}-\mathrm{C}_{5} \mathrm{H}_{4} M e\right), \quad-0.16 \quad\left(\left(\eta^{5}-\mathrm{C}_{5} \mathrm{H}_{4}\right) \mathrm{Si} \mathrm{Me}_{2}\left(\eta^{5}-\mathrm{C}_{5} \mathrm{H}_{4}\right)\right), \quad-3.15$ $\left(\left(\eta^{5}-\mathrm{C}_{5} \mathrm{H}_{4}\right) \mathrm{Si} \mathrm{Me}_{2} \mathrm{SiC}_{5} \mathrm{H}_{5}\right) . \quad M_{\mathrm{n}}=3.4 \times 10^{3}, M_{\mathrm{w}} / M_{\mathrm{n}}=1.18$.

The $\mathrm{C}_{5} \mathrm{H}_{5}$ end-capped oligomer $\mathbf{8 b}$ was obtained with $0.1 \mathrm{M} \mathrm{Li}\left[\mathrm{C}_{5} \mathrm{H}_{5}\right]$ in THF by similar procedure. Yield $61 \% .{ }^{1} \mathrm{H}$ NMR $\left(\mathrm{C}_{6} \mathrm{D}_{6}, 25^{\circ} \mathrm{C}\right): \delta 6.68\left(\mathrm{SiC}_{5} H_{5}\right), 6.58$ $\left(\mathrm{SiC}_{5} H_{5}\right), \quad 4.27 \quad\left(\left(\eta^{5}-\mathrm{C}_{5} H_{4}\right) \mathrm{Si}\left(\eta^{5}-\mathrm{C}_{5} H_{4}\right)\right), \quad 4.11 \quad\left(\left(\eta^{5}-\mathrm{C}_{5} H_{4}\right) \mathrm{Si}\left(\eta^{5}-\mathrm{C}_{5} H_{4}\right)\right), \quad 4.04$ $\left(\left(\eta^{5}-\mathrm{C}_{5} H_{4}\right) \mathrm{SiC}_{5} \mathrm{H}_{5}\right), \quad 3.48 \quad\left(\mathrm{SiC}_{5} H_{5} \quad\right.$ for $\left.\quad \mathrm{I}\right), \quad 2.99 \quad\left(\mathrm{SiC}_{5} H_{5} \quad\right.$ for $\left.\quad \mathrm{II}\right), \quad 0.55$ $\left(\left(\eta^{5}-\mathrm{C}_{5} \mathrm{H}_{4}\right) \mathrm{Si} \mathrm{Se}_{2}\left(\eta^{5}-\mathrm{C}_{5} \mathrm{H}_{4}\right)\right), 0.30\left(\left(\eta^{5}-\mathrm{C}_{5} \mathrm{H}_{4}\right) \mathrm{Si} M e_{2} \mathrm{C}_{5} \mathrm{H}_{5}\right), 0.14\left(\left(\eta^{5}-\mathrm{C}_{5} \mathrm{H}_{4}\right) \mathrm{Si} e_{2} \mathrm{C}_{5} \mathrm{H}_{5}\right)$. ${ }^{13} \mathrm{C}\left\{{ }^{1} \mathrm{H}\right\} \operatorname{NMR}\left(\mathrm{C}_{6} \mathrm{D}_{6}, 25^{\circ} \mathrm{C}\right): \delta 134.1\left(\mathrm{Si}_{5} \mathrm{H}_{5}\right), 131.0\left(\mathrm{SiC}_{5} \mathrm{H}_{5}\right), 74.0\left(\eta^{5}-C_{5} \mathrm{H}_{4}\right), 72.1$ $\left(\eta^{5}-C_{5} \mathrm{H}_{4}\right), 69.0\left(\eta^{5}-C_{5} \mathrm{H}_{5}\right), 53.6\left(\mathrm{SiC}_{5} \mathrm{H}_{5}\right),-0.16\left(\left(\eta^{5}-\mathrm{C}_{5} \mathrm{H}_{4}\right) \mathrm{Si}_{2} e_{2}\left(\eta^{5}-\mathrm{C}_{5} \mathrm{H}_{4}\right)\right),-3.12$ $\left(\left(\eta^{5}-\mathrm{C}_{5} \mathrm{H}_{4}\right) \mathrm{Si} e_{2} \mathrm{C}_{5} \mathrm{H}_{5}\right) . \quad M_{\mathrm{n}}=3.3 \times 10^{3}, M_{\mathrm{w}} / M_{\mathrm{n}}=1.35$.

Photolytic ROP of 1. A solution of $2.0 \mathrm{M} \mathrm{Na}\left[\mathrm{C}_{5} \mathrm{H}_{5}\right]$ in THF was added to $\mathbf{1}$ in 0.5 or $1 \mathrm{ml}$ of THF in the absence of light and irradiated at $5{ }^{\circ} \mathrm{C}$ with stirring. The solution colour became amber and the viscosity increased. The reaction was terminated by the addition of a few drops of degassed water. The amber PFS products $\mathbf{8 b}$ were isolated in yields of $76-93 \%$ by precipitation into hexanes and were dried under high vacuum (Table 1).

\section{References:}

(1) Pangborn, A. B.; Giardello, M. A.; Grubbs, R. H.; Rosen, R. K.; Timmers, F. J. Organometallics 1996, 15, 1518.

(2) Massey, J. A.; Kulbaba, K.; Winnik, M. A.; Manners, I. J. Polym. Sci., Part B: Polym. Phys. 2000, 38, 3032.

(3) Ni, Y.; Rulkens, R.; Manners, I. J. Am. Chem. Soc. 1996, 118, 4102.

(4) Ashe III, A. J. J. Am. Chem. Soc. 1970, 92, 1233. 